\title{
Thioester-Containing Protein-4 Regulates the Drosophila Immune Signaling and Function against the Pathogen Photorhabdus
}

\author{
Upasana Shokal Ioannis Eleftherianos \\ Department of Biological Sciences, Institute for Biomedical Sciences, The George Washington University, \\ Washington, D.C., USA
}

\section{Key Words}

Drosophila $\cdot$ Thioester-containing protein $\cdot$ Photorhabdus .

Immune signaling $\cdot$ Melanization

\begin{abstract}
Despite important progress in identifying the molecules that participate in the immune response of Drosophila melanogaster to microbial infections, the involvement of thioestercontaining proteins (TEPs) in the antibacterial immunity of the fly is not fully clarified. Previous studies mostly focused on identifying the function of TEP2, TEP3 and TEP6 molecules in the $D$. melanogaster immune system. Here, we investigated the role of TEP4 in the regulation and function of $D$. melanogaster host defense against 2 virulent pathogens from the genus Photorhabdus, i.e. the insect pathogenic bacterium Photorhabdus luminescens and the emerging human pathogen $P$. asymbiotica. We demonstrate that Tep4 is strongly upregulated in adult flies following the injection of Photorhabdus bacteria. We also show that Tep4 loss-of-function mutants are resistant to $P$. luminescens but not to $P$. asymbiotica infection. In addition, we find that inactivation of Tep4 results in the upregulation of the Toll and Imd immune pathways, and the downregulation of the Jak/Stat and Jnk pathways upon Photorhabdus infection. We document that loss of Tep4 promotes melanization and phenoloxidase
\end{abstract}

\section{KARGER}

๑) 2016 S. Karger AG, Basel

E-Mail karger@karger.com

www.karger.com/jin activity in the mutant flies infected with Photorhabdus. Together, these findings generate novel insights into the immune role of TEP4 as a regulator and effector of the D. melanogaster antibacterial immune response.

ㄷ) 2016 S. Karger AG, Basel

\section{Introduction}

A key innate immune defense against bacterial infection includes the activation of the complex complement system that involves the action of complement proteins. These proteins contain an unstable thioester bond and belong to the family of thioester-containing proteins (TEPs). Most TEPs share a 4-amino-acid sequence (CGEQ) that allows covalent bond formation with microbial surfaces and promotes opsonization [1]. Their function involves the recognition and phagocytosis of microbes that ultimately lead to their elimination from the host [2].

TEPs emerged early in evolution and are present in a wide variety of organisms [3]. TEPs have been found to participate in the mosquito immune response to certain bacteria and protozoa parasites $[4,5]$. For example, it was previously reported that the transcription of Tep1 in the African malaria mosquito Anopheles gambiae (aTep1) is upregulated upon infection with Escherichia coli and 
aTEP1 protein functions as an opsonin that promotes phagocytosis of $E$. coli and other Gram-negative bacteria [5]. It has also been shown that RNAi-mediated knock down of aTep1 prevents melanization of Plasmodium parasites [6]. Although there is sufficient information on the function of mosquito TEPs against Plasmodium parasites and bacteria, there are only a few studies on the potential antimicrobial immune role of TEPs in the insect model, Drosophila melanogaster $[1,7,8]$.

The D. melanogaster genome contains 6 Tep genes (Tep1-Tep6), of which Tep5 is a pseudogene and Tep6 or macroglobulin-complement related (MCR) lacks a functional thioester-binding site because of the substitution of cysteine residue by serine in the thioester motif [1]. TEP1-TEP4 proteins are constitutively expressed in plasmatocytes [1] whereas TEP6 has been shown to be a transmembrane protein that is required for septate junction formation [9]. Specifically, the Tep4 gene is expressed in both larval stages as well as in adult flies [1]. Other than plasmatocytes, it is induced in the fat body of the head and ventral side of the abdominal epidermis and spermatozoon $[1,10,11]$.

Few studies have examined the role of TEPs in the innate immune response of D. melanogaster. Previously, it has been shown that TEP2, TEP3 and TEP6 promote phagocytosis in Drosophila S2 cells after infection with the bacteria E. coli and Staphylococcus aureus or the fungus Candida albicans, respectively [8]. A recent study has also reported the high sensitivity of $D$. melanogaster Tep3 mutant larvae to infection with the nematode parasite Heterorhabditis bacteriophora [12]. While previous studies focused mostly on TEP2, TEP3 and TEP6 molecules, the involvement of TEP4 in the antimicrobial immune response of the fly is largely unexplored. Whole-genome expression profiles have indicated that Tep4 is differentially induced upon bacterial, fungal and parasitoid infection $[1,13,14]$. However, no changes were found in the survival response of Tep 4 mutant flies against infection with various bacterial and fungal pathogens [1]. It was concluded that the potential contribution of Tep genes in the $D$. melanogaster immune response could be better understood by using insect-specific pathogens, such as Photorhabdus bacteria.

Photorhabdus is a genus of entomopathogenic bacteria and a member of the family Enterobacteriaceae. In addition to being a highly virulent pathogen of insects, Photorhabdus maintains a mutualistic relationship with nematodes from the family Heterorhabditidae [15]. Here we have used two distinct species of Photorhabdus bacteria; $P$. luminescens, which is an obligate insect pathogen and
P. asymbiotica, which is able to infect both insects and humans [16]. The bacteria produce a wide range of toxins, virulence factors and hydrolytic enzymes $[17,18]$. Important genomic differences between these two Photorhabdus species include the presence of a plasmid related to PMT1 from Yersinia pestis together with novel pathogenicity islands in $P$. asymbiotica that converts this bacterium into a human pathogen. In addition, the presence of fewer diverse insecticidal genes than in P. luminescens allows $P$. asymbiotica to also persist as an insect pathogen $[19,20]$.

Here, we report the involvement of TEP4 in the innate immune response of the fly against $P$. luminescens and $P$. asymbiotica. We determine that transcription of Tep4 gene plays an essential role in the regulation of immune signaling during Photorhabdus infection and that interference with Tep4 gene transcription can affect the resistance and phenoloxidase (PO) activity in response to infection by these pathogens. These findings are important because they provide compelling evidence for the complexity and flexibility of the fly immune system.

\section{Materials and Methods}

Fly and Bacterial Strains

D. melanogaster fly strains $y w$ and the P-element insertion mutant tep4 (15936, Bloomington) were used for infections. Fly strains were maintained and amplified for experimentation with instant Drosophila media (Carolina Biological Supply) made in deionized water. All stocks were maintained at $25^{\circ} \mathrm{C}$ and a $12: 12$ hour light:dark photoperiod. Adult flies (7-10 days old) were used in all bacterial infection assays.

Bacterial strains P. luminescens subsp. laumondii (strain TT01), P. asymbiotica subsp. asymbiotica (strain ATCC 43949) and E. coli K12 were used for all fly infections. Bacterial cultures were prepared in sterile Luria-Bertani broth and grown for approximately $18-22 \mathrm{~h}$ at $30^{\circ} \mathrm{C}$ on a rotary shaker at $220 \mathrm{rpm}$. Bacterial cultures were pelleted down and were washed and resuspended in $1 \times$ sterile phosphate-buffered saline (PBS, Sigma Aldrich). Bacterial concentrations were adjusted to an optical density (OD, $600 \mathrm{~nm})$ of 0.1 for $P$. luminescens, 0.25 for $P$. asymbiotica and 0.015 for E. coli, using a spectrophotometer (NanoDropTM 2000c, Thermo Fisher Scientific).

\section{Infection Assays and Survival Experiment}

Flies were anesthetized with carbon dioxide and then injected in the thorax with $18.4 \mathrm{nl}(100-300 \mathrm{CFU})$ of each bacterial suspension (i.e. P. luminescens, P. asymbiotica or E. coli) or PBS (septic injury control) using a Nanoject II apparatus (Drummond Scientific) equipped with glass capillaries prepared with a micropipette puller (Sutter Instruments). Following injection, flies were transferred to fresh vials with instant media at $25^{\circ} \mathrm{C}$ and survival was scored at 6-hour intervals and up to $48 \mathrm{~h}$. Two replicates of 10 flies were used for each treatment and each assay was replicated 3 times. 


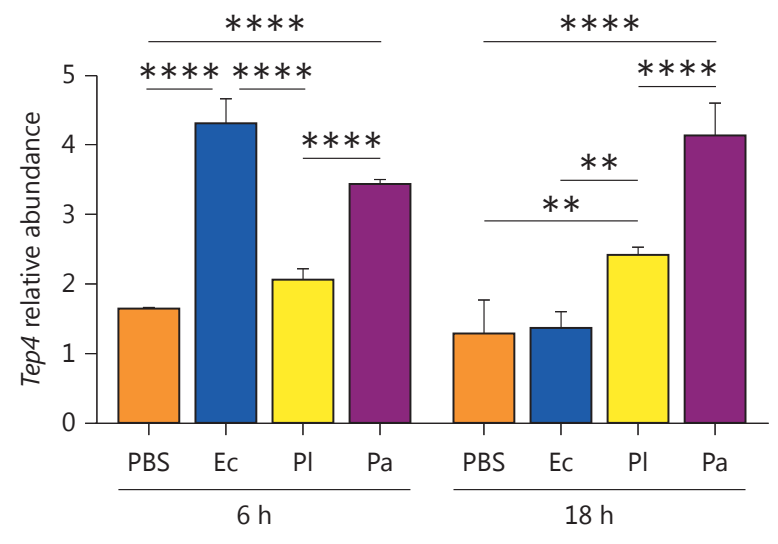

Fig. 1. Transcript levels of Tep4 in $y w$ flies following Photorhabdus infection. Transcript levels of Tep 4 in $y w$ flies at 6 and $18 \mathrm{~h}$ after injection with $\mathrm{PBS}$, E. coli (Ec), P. luminescens ( $\mathrm{Pl})$ or P. asymbiotica $(\mathrm{Pa})$ bacteria. Transcript levels are shown as relative abundance of transcripts normalized to RpL32 and are presented as the ratio between uninfected flies versus bacteria or PBS injected flies (3 individuals/experimental condition). ${ }^{* *} \mathrm{p}<0.01$, **** $\mathrm{p}<$ 0.0001 , significant differences. Bars show the means from 3 independent experiments and error bars represent standard deviations.

\section{Bacterial Load}

Four adult flies from each strain were injected with $P$. luminescens, $P$. asymbiotica or E. coli, and then frozen at 6 and $18 \mathrm{~h}$ after infection. DNA samples were extracted from the frozen flies using DNeasy blood and tissue kit (Qiagen). For bacterial load estimation, all DNA samples were adjusted to $500 \mathrm{ng}$. Quantitative PCRs were carried out in twin-tech, semiskirted, 96-well plates on a Mastercycler ${ }^{\circledR}$ ep realplex 2 . The cycling conditions for estimating the bacterial load were $50^{\circ} \mathrm{C}$ for $2 \mathrm{~min}, 95^{\circ} \mathrm{C}$ for $2 \mathrm{~min}, 40$ cycles of $95^{\circ} \mathrm{C}$ for $15 \mathrm{~s}$ and an annealing step of $61^{\circ} \mathrm{C}$ for $15 \mathrm{~s}$. All samples were run in technical duplicates and the experiments were repeated 3 times. Standard curves for each bacterium were used to estimate the bacterial load in infected flies as described before [21].

\section{Gene Transcription}

Three to four adult flies from each strain were injected with $P$. luminescens, P. asymbiotica, E. coli or PBS and frozen 6 and $18 \mathrm{~h}$ later. Total RNA was extracted using the PrepEase RNA spin kit (Affymetrix USB). Complementary DNA synthesis, quantitative RT-PCR (qRT-PCR) and analysis of the data were performed as previously described [22]. All primers used for the PCR assays are listed in online supplementary table S1 (for all online suppl. material, see www.karger.com/doi/10.1159/000450610). Data are presented as the ratio between uninfected flies versus bacteria- or PBS-injected flies.

\section{Hemolymph Extraction, PO Activity and Melanization}

For hemolymph collection, 20 flies were injected with bacteria or PBS as mentioned above, and PO activity was measured as de- scribed previously [23]. Briefly, at $3 \mathrm{~h}$ after injection, the flies were placed on a $10-\mu \mathrm{m}$ spin column (Pierce, Thermo Fisher) containing $30 \mu \mathrm{l}$ of $2.5 \times$ protease inhibitor (Sigma) and covered with five 4 -mm glass beads (VWR). These were centrifuged at $4{ }^{\circ} \mathrm{C}$ and $13,000 \mathrm{rpm}$ for $20 \mathrm{~min}$. Protein concentrations were then adjusted using a BCA test. A total volume of $40 \mu \mathrm{l}$ containing a mixture of $15 \mu \mathrm{g}$ of protein (diluted in $2.5 \times$ protease inhibitor) with $5 \mathrm{mM}$ $\mathrm{CaCl}_{2}$ was added to $160 \mu \mathrm{l}$ of L-DOPA solution (15 mM in phosphate buffer, $\mathrm{pH}$ 6.6). After $36 \mathrm{~min}$ of incubation at $29^{\circ} \mathrm{C}$ in the dark, the PO activity (OD $492 \mathrm{~nm}$ ) was measured for each sample against a blank control. Each experiment was performed in biological duplicate and repeated 3 times. Melanization spots on the site of injury were observed at $3 \mathrm{~h}$ after injection, using a Nikon SMZ18 stereomicroscope $(\times 10$ magnification) with Nikon EOS Rebel T3i camera.

\section{Statistical Analysis}

Statistics were performed using the GraphPad Prism6 software. Statistical analysis of data from survival experiments was conducted using the log-rank (Mantel-Cox) and $\chi^{2}$ tests. Unpaired twotailed Student's t test and one-way analysis of variance (ANOVA) with Tukey's post hoc test for multiple comparisons were used for analyzing bacterial load, gene expression data and PO activity results. $\mathrm{p}<0.05$ was considered statistically significant.

\section{Results}

\section{Tep4 Is Induced by Photorhabdus Infection}

We first examined the transcript levels of Tep4 in the untreated flies of the reference strain $(y w)$ and the loss-offunction tep 4 mutants. We found that transcript levels of Tep4 were significantly reduced in the mutants compared to the background reference strain (online suppl. fig. S1a). We then investigated the transcriptional regulation of Tep4 in background flies injected by either Photorhabdus sp., E. coli (nonpathogenic control) or PBS (septic injury control) at 6 and $18 \mathrm{~h}$ after injection. We observed that Tep4 was upregulated at $6 \mathrm{~h}$ after infection with $E$. coli and P. asymbiotica whereas after $18 \mathrm{~h}$, Tep4 was induced by the Photorhabdus bacteria only (fig. 1). We also noticed no changes in Tep4 gene transcript levels in tep 4 mutants upon bacterial infection or in the uninfected controls at any of the time points tested (online suppl. fig. $\mathrm{S} 1 \mathrm{~b})$. These results show that infection of $D$. melanogaster with the insect-specific pathogen $P$. luminescens or the related human pathogen $P$. asymbiotica results in significant upregulation of Tep4 in the adult fly.

\section{Tep4 Mutants Are Resistant to P. luminescens Infection}

To test whether inactivation of Tep4 affects the survival ability of the fly against infection by Photorhabdus pathogens, we tested the survival response of tep 4 loss-of- 


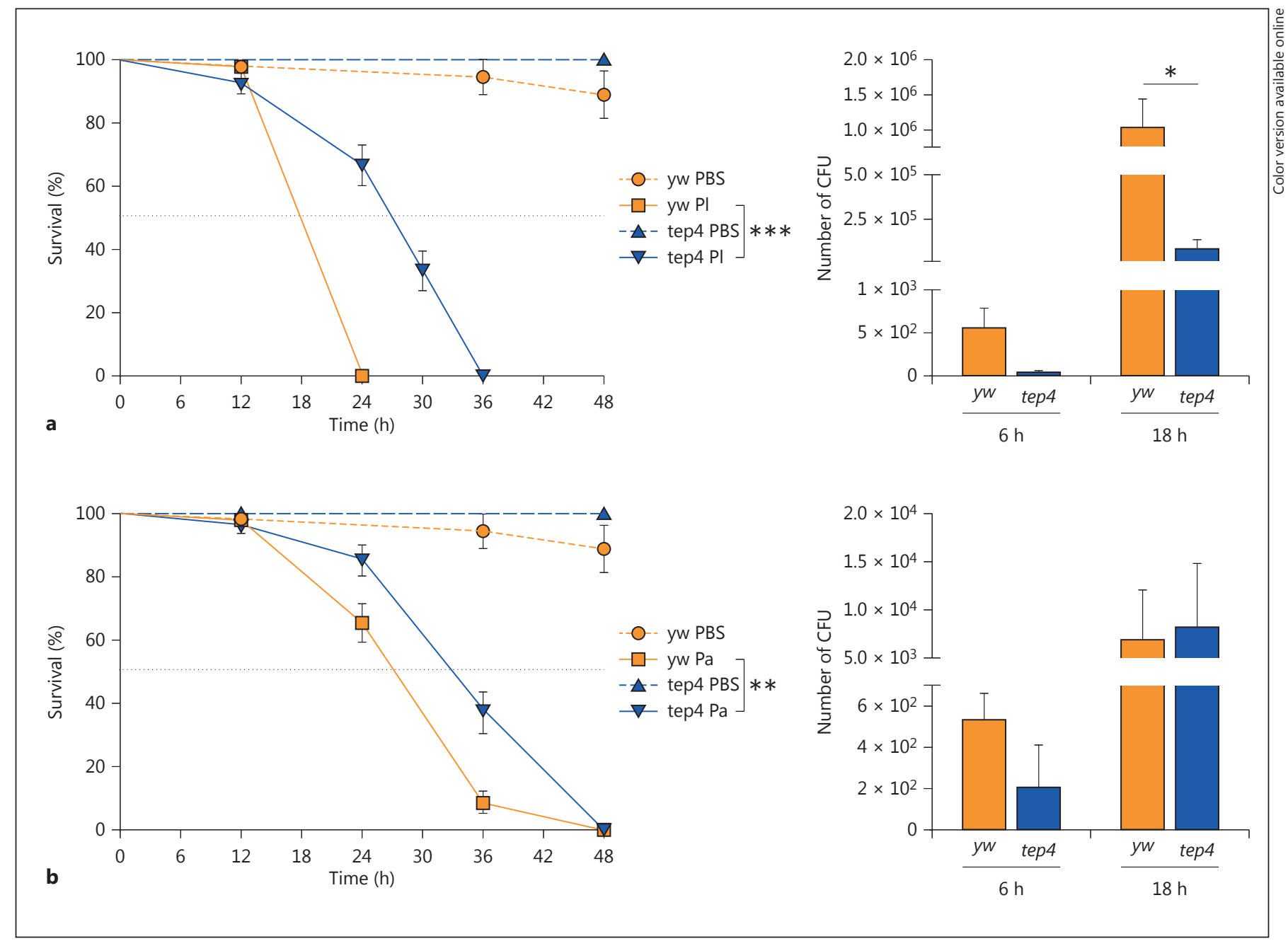

Fig. 2. Tep4 mutants are resistant to $P$. luminescens during the initial phases of infection. Survival of loss-of-function tep 4 mutants and control flies $(y w)$ after intrathoracic injection with $P$. luminescens (Pl) (a) or P. asymbiotica (Pa) (b) (20 flies/experimental condition). Injections with PBS served as injury control. Survival was monitored for $48 \mathrm{~h}$ at 6-hour intervals and the black dotted line represents $50 \%$ survival. CFU of $P$. luminescens (a) or $P$. asym-

function mutants and their background controls to infection by P. luminescens and P. asymbiotica. We found that tep 4 mutants and control flies succumbed within $36 \mathrm{~h}$ of $P$. luminescens infection and within $48 \mathrm{~h}$ of $P$. asymbiotica infection. We noticed that after $24 \mathrm{~h}$, tep4 mutants had survived significantly better ( $67 \%$ were alive) than the control flies ( $0 \%$ were alive) (fig. 2a). Likewise, $85 \%$ of tep 4 mutants and $65 \%$ of the controls were alive $24 \mathrm{~h}$ after P. asymbiotica infection (fig. 2b). Thirty-six hours after infection with $P$. asymbiotica, $37 \%$ of tep 4 mutant flies were alive versus $8 \%$ of the controls (fig. $2 \mathrm{~b}$ ). We further biotica (b) at 6 and $18 \mathrm{~h}$ after injection were determined by quantitative PCR of mcf-1 (P. luminescens) and tccC3 (P. asymbiotica) in tep 4 mutants and their background control $y w$ flies (5 individuals/experimental condition). ${ }^{*} \mathrm{p}<0.05,{ }^{* *} \mathrm{p}<0.01,{ }^{* * *} \mathrm{p}<0.001$, significant differences. The means from 3 independent experiments are shown and error bars represent standard errors (survival) and standard deviation (bacterial load).

observed that injection with nonpathogenic E. coli or sterile PBS did not affect the survival of tep4 mutant flies or their background controls (online suppl. fig. S2a). These results indicate that inactivation of Tep4 provides a survival advantage to $D$. melanogaster flies in response to infection with $P$. luminescens and $P$. asymbiotica pathogenic bacteria.

To investigate whether increased survival of tep4 lossof-function mutants to Photorhabdus infection is due to the decreased persistence of the bacteria in the infected flies, we estimated bacterial load during the course of the 
infection. For this, we evaluated the number of CFU by quantitative PCR by amplifying the genes $m c f-1$ and $t c c C 3$ in P. luminescens and P. asymbiotica [24, 25], respectively, at 6 and $18 \mathrm{~h}$ after infection. Previous studies have shown that the levels of virulence factors produced by Photorhab$d u s$ do not change during insect infection [26]. We observed that tep 4 mutants contained 12 times fewer numbers of $P$. luminescens $\mathrm{CFU}$ compared to the background controls at $18 \mathrm{~h}$ after infection (fig. 2a), but there were no significant differences in P. asymbiotica CFU between the 2 fly strains (fig. 2b). Also, tep 4 mutants and their controls contained similar numbers of $E$. coli cells (online suppl. fig. S2b). These results indicate that deficiency in Tep4 confers resistance to $P$. luminescens infection only.

\section{Functional Tep4 Is Essential for Immune Signaling}

Activation upon Photorhabdus Infection

The hallmark of the D. melanogaster host defense is the definition of 2 major signaling pathways, Toll and Immune deficiency (Imd). The Toll pathway is reminiscent of the IL1/TLR4 Myd-88 dependent pathway whereas the Imd pathway presents striking similarities with the TNFR and TLR4 MyD88-independent pathways [27, 28]. These pathways activate different members of the NF- $\kappa B$ family of transcription factors, and induce the expression of several target genes including those encoding antimicrobial peptides (AMP) [29, 30]. The Toll pathway is induced by Lys-type peptidoglycan present on most Gram-positive bacteria, while the Imd pathway is triggered by diaminopimelic acid-type peptidoglycan that composes the cell wall of Gram-negative and some Gram-positive bacteria [31]. The Janus kinase/signal transducer and activator of transcription (Jak/Stat) and c-Jun N-terminal kinase (Jnk) signaling pathways can also act in either competing or cooperative modes to regulate immune effector genes in the fly $[32,33]$. To investigate whether the increased survival of Tep4 mutant flies to Photorhabdus infection could also be due to increased immune signaling activation, we examined the transcriptional activation of Toll, Imd, JAK/STAT and JNK immune pathways in tep 4 lossof-function mutant flies infected with the bacteria. We first estimated the transcript levels of the AMP Defensin as a bacterial-specific readout of the Toll pathway [29]. We have previously shown that infection of wild-type flies with Photorhabdus induces low levels of Defensin $[21,22]$. Therefore, we asked whether Tep4 inactivation can alter the transcript levels of Defensin in response to Photorhabdus. We observed that Defensin was slightly upregulated in $y w$ flies at $6 \mathrm{~h}$ after infection with any of the 3 bacteria (fig. 3a; online suppl. fig. S3a). Interesting- ly, there was an early (at $6 \mathrm{~h}$ ) transcriptional activation of Defensin in tep4 mutants upon infection with P. luminescens or $P$. asymbiotica, but not with nonpathogenic E. coli (fig. 3a; online suppl. fig. S3a). Then, we evaluated Imd pathway activation by estimating the transcript levels of the Cecropin-A1 (AMP) gene in infected flies [30, 34]. We observed significantly higher transcript levels of Cecropin- $A 1$ in tep 4 mutants than in their $y w$ background controls following infection for $18 \mathrm{~h}$ with Photorhabdus (fig. 3b). These results indicate that inactivation of Tep4 results in the activation of Toll and Imd signaling in response to Photorhabdus infection.

We then examined the activation of Jak/Stat and Jnk signaling pathways in tep 4 mutant and their background controls, by assessing the transcript levels of Turandot- $M$ (Tot-M) and Puckered (Puc) $[35,36]$. We noticed that Tot- $M$ was upregulated in $y w$ flies at $6 \mathrm{~h}$ after infection with either Photorhabdus species or E. coli (fig. 3c; online suppl. fig. S3c). We then observed that in tep4 mutant flies, Tot- $M$ was not upregulated at any time point after bacterial infection compared to background controls (fig. 3c; online suppl. fig. S3c). Also, Puc was significantly upregulated in control flies at $18 \mathrm{~h}$ after infection with Photorhabdus bacteria, but not with E. coli (fig. $3 \mathrm{~d}$; online suppl. fig. S3d). Strikingly, transcript levels of $P u c$ in tep4 mutants were significantly lower than in control flies upon infection with $P$. asymbiotica or $P$. luminescens (fig. 3d). These results indicate that activity of Tep4 is important for the regulation of Jak/Stat and Jnk signaling in D. melanogaster adult flies upon infection with the pathogen Photorhabdus.

Inactivation of Tep4 Increases the Melanization and PO Response to Photorhabdus Infection

We also asked whether inactivation of Tep4 affects PO activity and the melanization response, which form rapid immune reactions of the fly against microbial intruders [23]. Since the formation of a black spot around the injection site represents the output of the melanization reaction [37], here we first examined the in vivo melanization in tep 4 mutants and their control flies by visually inspecting the wound site at $3 \mathrm{~h}$ after injection with Photorhabdus sp., E. coli or PBS. We noticed the development of dark melanin spots in tep 4 mutants in response to bacteria, but $y w$ flies developed fainter spots after challenge with bacteria (fig. 4a; online suppl. fig. S4a).

To quantify the level of $\mathrm{PO}$ enzyme activity in infected and uninfected tep 4 mutants and their background controls, we extracted hemolymph plasma from the flies at $3 \mathrm{~h}$ after injection with bacteria or buffer alone. We found 


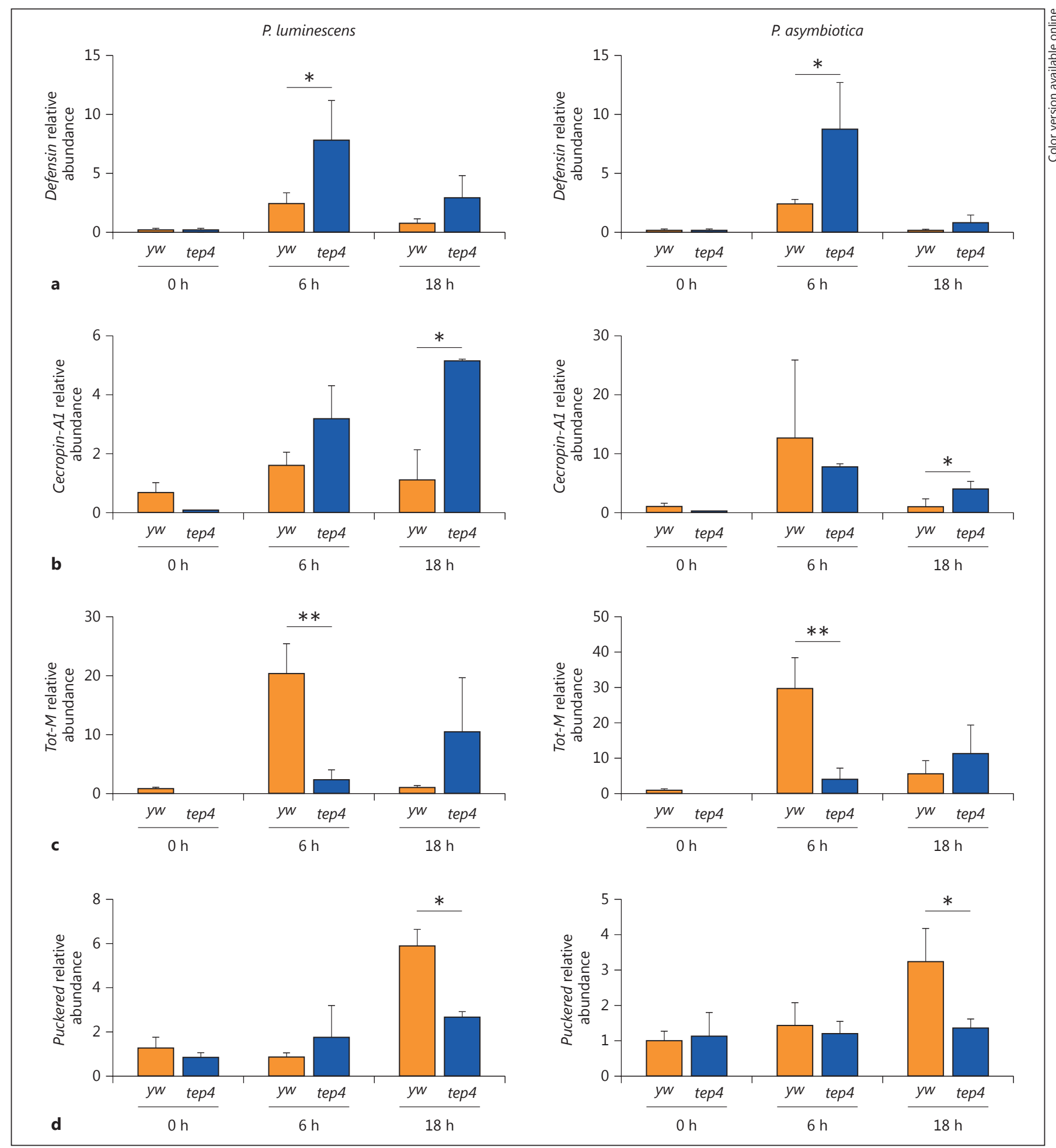

Fig. 3. Tep4 modulates the activation of immune signaling in response to Photorhabdus. Transcript levels for Defensin (Toll pathway, a), Cecropin-A1 (Imd pathway, b), Tot-M (Jak/Stat pathway, c) and Puckered (Jnk pathway, d) in tep4 mutant and background control flies $(y w)$ at 0,6 and $18 \mathrm{~h}$ after infection with $P$. luminescens or $P$. asymbiotica ( $\mathrm{n}=3$ individuals per experimental condition).

Gene transcript levels are shown as relative abundance of transcripts normalized to $R p L 32$ and expressed as a ratio compared to flies injected with sterile PBS (negative control). Values represent the means from 3 biological replicates, and error bars represent standard deviations. ${ }^{*} \mathrm{p}<0.05,{ }^{* *} \mathrm{p}<0.01$, significant differences. 
Fig. 4. Melanization response and PO activity are elevated in tep 4 mutants upon Photorhabdus infection. a Melanization of the wound site in tep4 loss-of-function mutant flies and their background control strain $(y w)$ is shown $3 \mathrm{~h}$ after injection with PBS, $P$. luminescens or P. asymbiotica. Arrows indicate the site of injury. $\times 10$. b PO activity in the hemolymph plasma of tep4 mutants and control flies ( $y w$ ) $3 \mathrm{~h}$ after injection with $\mathrm{PBS}, P$. luminescens $(\mathrm{Pl})$ or $P$. asymbiotica ( $\mathrm{Pa})(\mathrm{n}=20$ flies), as measured by the OD at $492 \mathrm{~nm}$ after incubation with L-DOPA. Values represent the means from 3 biological replicates and error bars represent standard deviations. ${ }^{* * *} \mathrm{p}<0.001$, significant differences.
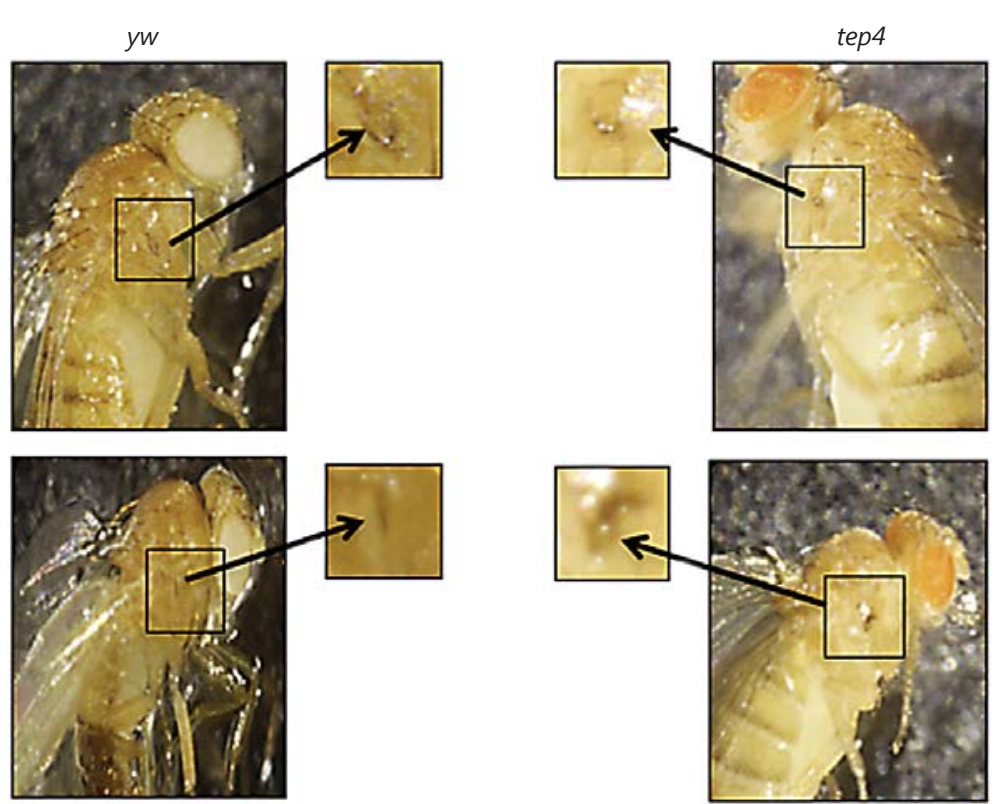

$\stackrel{\infty}{a}$

a
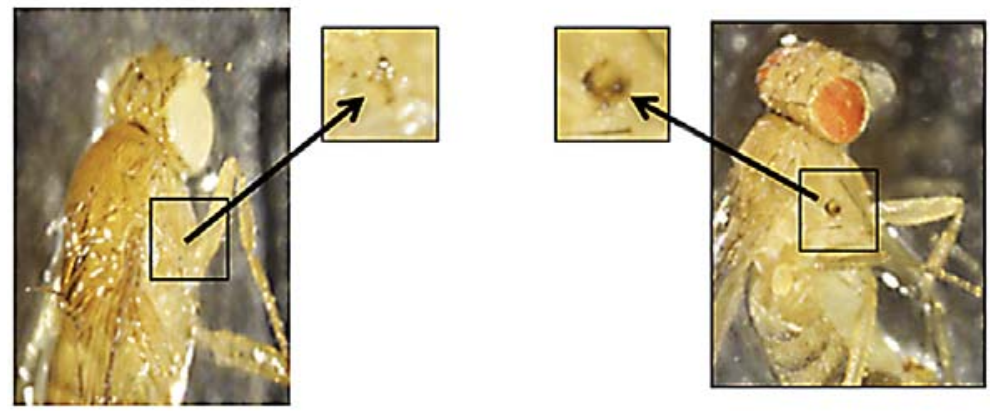

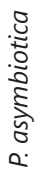

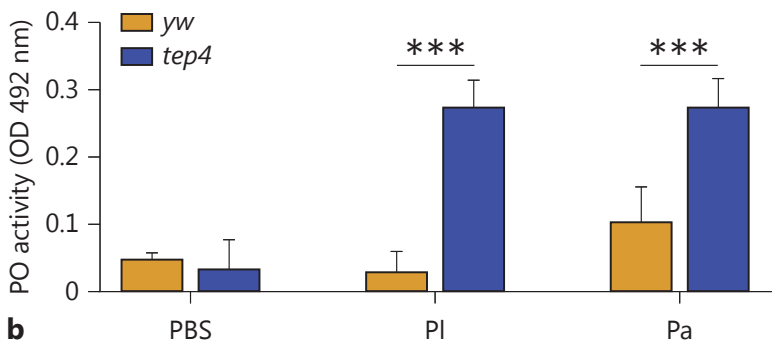

no significant changes in PO activity between tep4 mutant flies and $y w$ controls injected with PBS (fig. 4b). However, tep 4 mutants infected by either Photorhabdus sp. or E. coli displayed significantly higher PO activity levels than $y w$ flies (fig. 4b; online suppl. fig. S4b). These results imply that the absence of functional Tep4 in $D$. melanogaster adult flies promotes PO activity and the melanization response against infection with pathogenic Photorhabdus or nonpathogenic E. coli bacteria.

\section{Discussion}

Significant advances have been made recently in the field of Drosophila immunity; however, our understanding of the role of TEP molecules in the fly immune response remains incomplete $[1,30]$. Here, we have investigated the participation of Tep4 in the immune activity of $D$. melanogaster against two species of the virulent pathogen Photorhabdus. Although D. melanogaster has 
not been shown thus far to be a natural host for Photorhabdus, we and others have recently started to exploit the powerful tools of the fly to identify the molecules potentially involved in the interaction between the fly immune system and pathogenic microbes [22, 38, 39]. Photorhabdus bacteria express multiple pathogenicity factors with different specificities against a diverse range of insect species and tissues [40, 41], so we hypothesized that the response of D. melanogaster to Photorhabdus bacteria would be sufficiently different from other classes of bacteria that have been studied in this system [30].

Previous transcriptomic analyses have shown that Tep2 and Tep4 are upregulated in whole flies following infection with a mix of E. coli and Micrococcus luteus bacteria $[1,42,43]$. Here, we showed that Tep4 is transcriptionally activated by $P$. luminescens and $P$. asymbiotica infection, which implies that $D$. melanogaster responds differently to specific pathogens. Our finding that Tep4 is upregulated upon $E$. coli challenge is in accordance with the previous studies $[1,42]$. The upregulation of Tep4 in D. melanogaster by Photorhabdus bacteria prompted us to speculate that certain TEP molecules might play an important role in the interaction between the fly immune response and Photorhabdus. The outcome of this interaction could involve changes in the sensitivity of the flies upon infection with these pathogens due to altered immune signaling or function. We have found that loss of Tep4 delays the mortality of mutant flies during the initial and intermediate stages of infection with $P$. luminescens or $P$. asymbiotica. Consequently, inactivation of Tep4 partially promotes the survival of the fly against Photorhabdus; however, the protective effect is not complete because the mutants eventually succumb to infection with the pathogens. Lack of changes in the sensitivity of tep4 mutants to nonpathogenic E. coli infection indicates that the protective effect is predominantly or exclusively conferred to the pathogens. Similar to our findings, a previous study also reported increased survival of hemizygous tep4 mutants towards bacterial infection [1]; however, another study has shown tep4 mutants to be sensitive to infection with the bacterium Porphyromonas gingivalis [7].

Given that Photorhabdus is not a natural pathogen of $D$. melanogaster, the positive effect of Tep4 gene inactivation on the survival of the mutant flies is probably specific to these pathogens as well as to as-of-yet unidentified natural pathogens of $D$. melanogaster with a mode of action similar to Photorhabdus [17, 20]. Such natural pathogens may employ distinct strategies to interact with the D. melanogaster immune system as a result of antagonis- tic coevolution with the fly. This assumption is supported by a previous study which examined the molecular evolution of innate immune system components in D. melanogaster, and proposed that certain Tep genes might have specific functions against distinct pathogens as a result of their rapid evolution due to strong positive selection forces that might act on these molecules [44]. An alternative explanation for the increased survival of tep4 mutants could be that upregulation of Tep4 in flies infected with certain pathogens, such as Photorhabdus, result in inflammatory responses that severely affect the survival of the flies during the first few hours of infection and eventually lead to insect death. Lack of changes in the P. asymbiotica burden between tep 4 mutants and control flies imply the involvement of other immune mechanisms that could promote survival upon infection with this pathogen.

There is a previous indication that Photorhabdus can interact with $A M P$ gene expression in the fly [22], and that ligands produced by hemocytes could induce immune signaling in certain tissues (e.g. the fat body) that could, in turn, affect the activation of Tep genes [32]. The effect of Tep4 gene inactivation on the increased survival of mutant flies with Photorhabdus infection formed the basis for testing whether Tep4 activity plays a regulatory role in the $D$. melanogaster immune system. For this, we examined whether a loss of Tep4 can affect the outcome of immune signaling in response to Photorhabdus. Unexpectedly, Toll and Imd pathway activation was consistently affected in the tep 4 mutant flies following $P$. luminescens or P. asymbiotica infection. Similarly, Jak/Stat and Jnk pathways were significantly impaired in the tep4 mutants upon Photorhabdus infection. Our results strongly indicate that normal transcription of Tep4 is required for the efficient activation of immune signaling in D. melanogaster flies responding to Photorhabdus. Since TEP4 is a secreted molecule [45], it may interact with the hemocytes and fat-body cells in a distinct manner when Photorhabdus bacteria are present in the fly hemocoel. Such interactions could then lead to changes in the regulation of genes that are induced in these tissues and the expression of which is controlled by specific immune signaling pathways. Therefore changes in immune signaling in Photorhabdus-infected flies, in which Tep4 gene expression is compromised, potentially lead to the production of molecules (presumably AMP) that provide protection to the pathogens during the early or intermediate stages of infection.

The proPO system is a rapid and efficient antimicrobial immune reaction that links humoral and cellular im- 
Fig. 5. Proposed model for the participation of TEP4 in the immune response of D. melanogaster against the pathogen Photorhabdus. 1. Tep4 is upregulated upon Photorhabdus infection. 2. Upregulation of Tep4 reduces Toll and Imd signaling in response to Photorhabdus infection. 3. Upregulation of Tep4 is required for the activation of Jak/Stat and Jnk signaling against Photorhabdus. 4. Regulation of immune signaling pathways by Tep 4 induction leads to differential induction of AMP-encoding genes and stress-related genes following infection with Photorhabdus. 5. During the early stages of Photorhabdus infection, upregulation of Tep4 decreases the PO activity and melanization response. 6 . Decrease in the activity of $\mathrm{PO} /$ melanization and interference with innate immune signaling alters the survival response of adult flies to Photorhabdus infection.

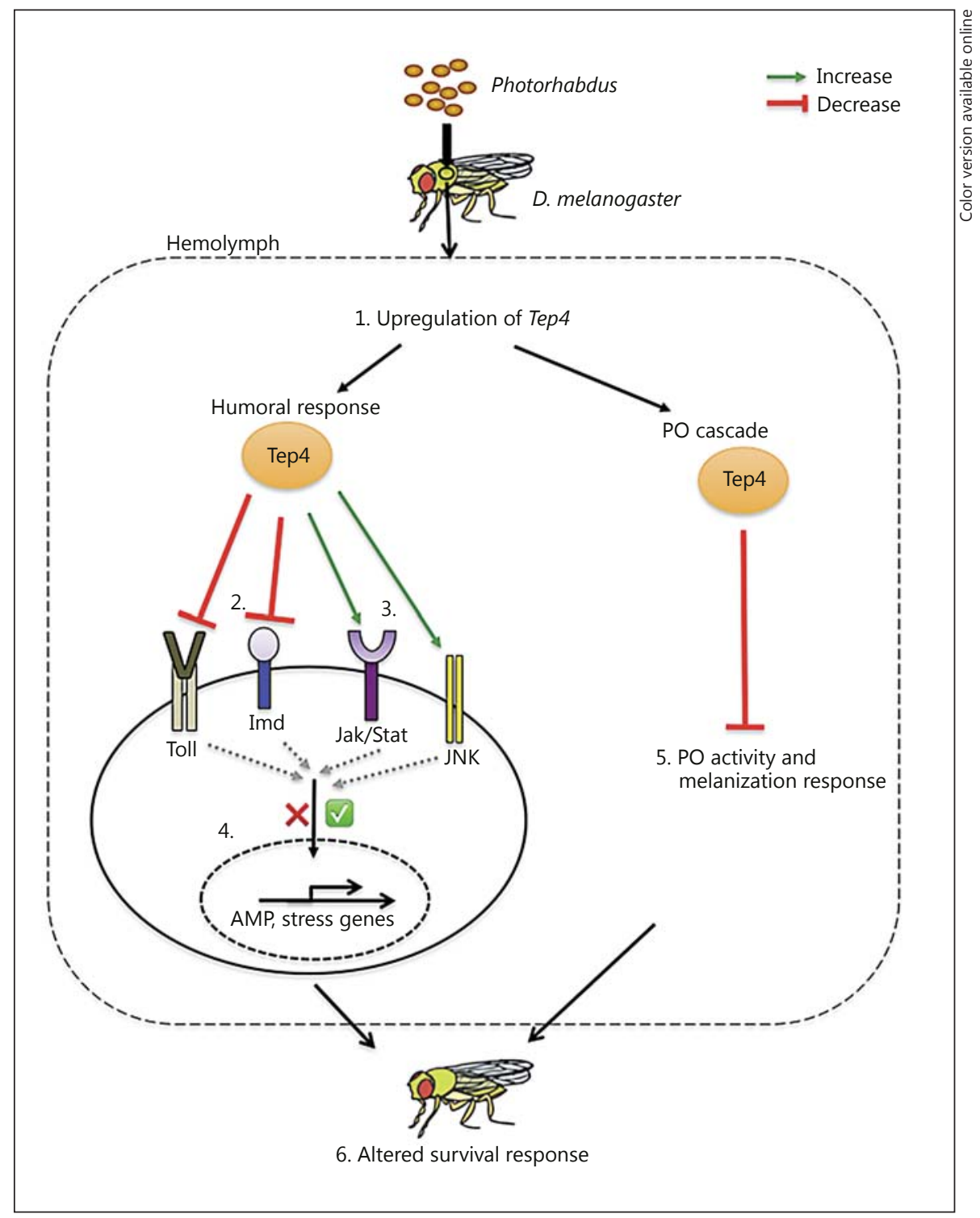

munity [46]. Previously, it has been proposed that during infection, TEP molecules in the hemolymph can interact with the fat body (and possibly with other tissues) to activate signaling pathways that regulate immune functions against the invading microbes [45]. Therefore, we tested whether infection with Photorhabdus could influence PO activity in the background control flies and whether inactivation of Tep 4 can further affect PO activity in the mutants infected with the pathogens. We were not surprised to find that Photorhabdus infection reduces $\mathrm{PO}$ activity in the hemolymph as well as the melanization response because both $P$. luminescens and $P$. asymbiotica employ mechanisms to interfere with the activation of the insect
proPO cascade [47-50]. Our results further reveal high levels of PO in tep4 mutants infected with either Photorhabdus pathogen. Because melanization and activation of the proPO system are rapid immune responses, these results could also explain the increased persistence of Photorhabdus bacteria in tep4 mutant flies over time. We suspect that high PO activity in tep4 mutant flies reduces bacterial load and, as a result, improves survival during the early phase of infection. Furthermore, these findings show, for the first time, an apparent link between tep 4 transcriptional gene expression and PO activity. Interestingly, this effect is not restricted only to Photorhab$d u s$ infections, but also to infections with nonpathogenic 
E. coli bacteria. This is an important finding because it shows that the proPO system in insects can be regulated by pathogens in order to evade the host immune system, but also by host molecules that can markedly affect immune signaling and microbial resistance and consequently influence the outcome of the infection.

Taken together, we have shown that Tep4 can modulate the immune response of $D$. melanogaster adult flies against infection with the virulent pathogens $P$. luminescens and P. asymbiotica. This can be attributed to its critical role as a regulator of immune signaling and function in response to these pathogens (fig. 5). It is currently unknown whether the expression of TEPs in the fly can be activated by proteases produced by pathogenic microbes or by the host [44]. Given that Photorhabdus bacteria secrete a battery of proteases [51], our future efforts will focus on exploring their association with Tep4 immune activity and the participation of the latter in cellular immune function of the fly against Photorhabdus and their cognate nematode partners [15]. Of note, certain tep fly mutants were recently shown to be sensitive to infection by entomopathogenic nematodes when compared to background controls [12]. We expect that such studies will unravel the regulatory role and specific function of TEP molecules in the antipathogen immune response of the fly and probably that of other invertebrate animals.

\section{Acknowledgements}

We thank members of the Department of Biological Sciences at GWU for critical reading of the manuscript. This research was supported by a start-up fund from the Columbian College of Arts and Sciences at GWU to I.E., the Harlan Summer Fellowships from the Department of Biological Sciences at GWU to U.S. and a scholarship from the Cosmos Club Foundation (Washington, D.C.) to U.S.

\section{Disclosure Statement}

The authors declare no competing interests.

\section{References}

1 Bou Aoun R, Hetru C, Troxler L, Doucet D, Ferrandon D, Matt N: Analysis of thioestercontaining proteins during the innate immune response of Drosophila melanogaster. J Innate Immun 2011;3:52-64.

2 Merle NS, Church SE, Fremeaux-Bacchi V, Roumenina LT: Complement system. Part I. Molecular mechanisms of activation and regulation. Front Immunol 2015;6:262.

3 Blandin S, Levashina EA: Thioester-containing proteins and insect immunity. Mol Immunol 2004;40:903-908.

4 Levashina EA, Moita LF, Blandin S, Vriend G, Lagueux M, Kafatos FC: Conserved role of a complement-like protein in phagocytosis revealed by dsRNA knockout in cultured cells of the mosquito, Anopheles gambiae. Cell 2001;104:709-718.

5 Baxter RH, Chang CI, Chelliah Y, Blandin S, Levashina EA, Deisenhofer J: Structural basis for conserved complement factor-like function in the antimalarial protein TEP1. Proc Natl Acad Sci USA 2007;104:11615-11620.

6 Blandin S, Shiao SH, Moita LF, Janse CJ, Waters AP, Kafatos FC, Levashina EA: Complement-like protein TEP1 is a determinant of vectorial capacity in the malaria vector Anopheles gambiae. Cell 2004;116:661-670.

7 Igboin CO, Tordoff KP, Moeschberger ML, Griffen AL, Leys EJ: Porphyromonas gingivalis-host interactions in a Drosophila melanogaster model. Infect Immun 2011;79:449-458.

8 Stroschein-Stevenson SL, Foley E, O'Farrell $\mathrm{PH}$, Johnson AD: Identification of Drosophila gene products required for phagocytosis of Candida albicans. PLoS Biol 2006;4:e4.

9 Batz T, Forster D, Luschnig S: The transmembrane protein macroglobulin complementrelated is essential for septate junction formation and epithelial barrier function in Drosophila. Development 2014;141:899-908.

10 Aradska J, Bulat T, Sialana FJ, Birner-Gruenberger R, Erich B, Lubec G: Gel-free mass spectrometry analysis of Drosophila melanogaster heads. Proteomics 2015;15:3356-3360.

11 Wasbrough ER, Dorus S, Hester S, HowardMurkin J, Lilley K, Wilkin E, Polpitiya A, Petritis K, Karr TL: The Drosophila melanogaster sperm proteome-II (DmSP-II). J Proteomics 2010;73:2171-2185.

12 Arefin B, Kucerova L, Dobes P, Markus R, Strnad H, Wang Z, Hyrsl P, Zurovec M, Theopold U: Genome-wide transcriptional analysis of Drosophila larvae infected by entomopathogenic nematodes shows involvement of complement, recognition and extracellular matrix proteins. J Innate Immun 2014;6:192204.

13 Wertheim B, Kraaijeveld AR, Schuster E, Blanc E, Hopkins M, Pletcher SD, Strand MR, Partridge L, Godfray HC: Genome-wide gene expression in response to parasitoid attack in Drosophila. Genome Biol 2005;6:R94.

14 Levy F, Rabel D, Charlet M, Bulet P, Hoffmann JA, Ehret-Sabatier L: Peptidomic and proteomic analyses of the systemic immune response of Drosophila. Biochimie 2004;86: 607-616.
15 Waterfield NR, Ciche T, Clarke D: Photorhabdus and a host of hosts. Annu Rev Microbiol 2009;63:557-574.

16 Costa SC, Chavez CV, Jubelin G, Givaudan A, Escoubas JM, Brehelin M, Zumbihl R: Recent insight into the pathogenicity mechanisms of the emergent pathogen Photorhabdus asymbiotica. Microbes Infect 2010;12:182-189.

17 Eleftherianos I, ffrench-Constant RH, Clarke DJ, Dowling AJ, Reynolds SE: Dissecting the immune response to the entomopathogen Photorhabdus. Trends Microbiol 2010;18: 552-560.

18 Eleftherianos IG: Novel antibiotic compounds produced by the insect pathogenic bacterium photorhabdus. Recent Pat Antiinfect Drug Discov 2009;4:81-89.

19 Wilkinson P, Paszkiewicz K, Moorhouse A, Szubert JM, Beatson S, Gerrard J, Waterfield NR, Ffrench-Constant RH: New plasmids and putative virulence factors from the draft genome of an Australian clinical isolate of Photorhabdus asymbiotica. FEMS Microbiol Lett 2010;309:136-143.

20 Wilkinson P, Waterfield NR, Crossman L, Corton C, Sanchez-Contreras M, Vlisidou I, Barron A, Bignell A, Clark L, Ormond D, Mayho M, Bason N, Smith F, Simmonds M, Churcher C, Harris D, Thompson NR, Quail M, Parkhill J, Ffrench-Constant RH: Comparative genomics of the emerging human pathogen Photorhabdus asymbiotica with the insect pathogen Photorhabdus luminescens. BMC Genomics 2009;10:302. 
21 Shokal U, Yadav S, Atri J, Accetta J, Kenney E, Banks K, Katakam A, Jaenike J, Eleftherianos I: Effects of co-occurring Wolbachia and Spiroplasma endosymbionts on the Drosophila immune response against insect pathogenic and non-pathogenic bacteria. BMC Microbiol 2016;16:16

22 Castillo JC, Shokal U, Eleftherianos I: Immune gene transcription in Drosophila adult flies infected by entomopathogenic nematodes and their mutualistic bacteria. J Insect Physiol 2013;59:179-185.

23 Binggeli O, Neyen C, Poidevin M, Lemaitre B: Prophenoloxidase activation is required for survival to microbial infections in Drosophila. PLoS Pathog 2014;10:e1004067.

24 Daborn PJ, Waterfield N, Silva CP, Au CP, Sharma S, Ffrench-Constant RH: A single Photorhabdus gene, makes caterpillars floppy ( $m c f)$, allows Escherichia coli to persist within and kill insects. Proc Natl Acad Sci USA 2002; 99:10742-10747.

25 Waterfield N, Hares M, Yang G, Dowling A, ffrench-Constant R: Potentiation and cellular phenotypes of the insecticidal toxin complexes of Photorhabdus bacteria. Cell Microbiol 2005;7:373-382.

26 Daborn PJ, Waterfield N, Blight MA, FfrenchConstant RH: Measuring virulence factor expression by the pathogenic bacterium Photorhabdus luminescens in culture and during insect infection. J Bacteriol 2001;183:58345839.

27 Lindsay SA, Wasserman SA: Conventional and non-conventional Drosophila Toll signaling. Dev Comp Immunol 2014;42:16-24.

28 Georgel P, Naitza S, Kappler C, Ferrandon D, Zachary D, Swimmer C, Kopczynski C, Duyk G, Reichhart JM, Hoffmann JA: Drosophila immune deficiency (IMD) is a death domain protein that activates antibacterial defense and can promote apoptosis. Dev Cell 2001;1: 503-514.

29 Imler JL, Bulet P: Antimicrobial peptides in Drosophila: structures, activities and gene regulation. Chem Immunol Allergy 2005;86: $1-21$.

30 Lemaitre B, Hoffmann J: The host defense of Drosophila melanogaster. Annu Rev Immunol 2007;25:697-743.
31 Tanji T, Hu X, Weber AN, Ip YT: Toll and IMD pathways synergistically activate an innate immune response in Drosophila melanogaster. Mol Cell Biol 2007;27:4578-4588.

32 Agaisse H, Perrimon N: The roles of JAK/ STAT signaling in Drosophila immune responses. Immunol Rev 2004;198:72-82.

33 Delaney JR, Stoven S, Uvell H, Anderson KV, Engstrom Y, Mlodzik M: Cooperative control of Drosophila immune responses by the JNK and NF-kappaB signaling pathways. EMBO J 2006;25:3068-3077.

34 Kylsten P, Samakovlis C, Hultmark D: The cecropin locus in Drosophila; a compact gene cluster involved in the response to infection. EMBO J 1990;9:217-224.

35 Brun S, Vidal S, Spellman P, Takahashi K, Tricoire H, Lemaitre B: The MAPKKK Mekk1 regulates the expression of Turandot stress genes in response to septic injury in Drosophila. Genes Cells 2006;11:397-407.

36 McEwen DG, Peifer M: Puckered, a Drosophila MAPK phosphatase, ensures cell viability by antagonizing JNK-induced apoptosis. Development 2005;132:3935-3946.

37 Tang H: Regulation and function of the melanization reaction in Drosophila. Fly (Austin) 2009;3:105-111.

38 Aymeric JL, Givaudan A, Duvic B: Imd pathway is involved in the interaction of Drosophila melanogaster with the entomopathogenic bacteria, Xenorhabdus nematophila and Photorhabdus luminescens. Mol Immunol 2010;47:2342-2348.

39 Vlisidou I, Waterfield N, Wood W: Elucidating the in vivo targets of Photorhabdus toxins in real-time using Drosophila embryos. Adv Exp Med Biol 2012;710:49-57.

40 ffrench-Constant RH, Dowling A, Waterfield NR: Insecticidal toxins from Photorhabdus bacteria and their potential use in agriculture. Toxicon 2007;49:436-451.

41 Rodou A, Ankrah DO, Stathopoulos C: Toxins and secretion systems of Photorhabdus luminescens. Toxins (Basel) 2010;2:1250-1264.

42 Lagueux M, Perrodou E, Levashina EA, Capovilla M, Hoffmann JA: Constitutive expression of a complement-like protein in toll and JAK gain-of-function mutants of Drosophila. Proc Natl Acad Sci USA 2000;97:1142711432.
43 Irving P, Troxler L, Heuer TS, Belvin M, Kopczynski C, Reichhart JM, Hoffmann JA, Hetru C: A genome-wide analysis of immune responses in Drosophila. Proc Natl Acad Sci USA 2001;98:15119-15124.

44 Jiggins FM, Kim KW: Contrasting evolutionary patterns in Drosophila immune receptors. J Mol Evol 2006;63:769-780.

45 Blandin SA, Wang-Sattler R, Lamacchia M, Gagneur J, Lycett G, Ning Y, Levashina EA, Steinmetz LM: Dissecting the genetic basis of resistance to malaria parasites in Anopheles gambiae. Science 2009;326:147-150.

46 Eleftherianos I, Revenis C: Role and importance of phenoloxidase in insect hemostasis. J Innate Immun 2011;3:28-33.

47 Eleftherianos I, Millichap PJ, ffrench-Constant RH, Reynolds SE: RNAi suppression of recognition protein mediated immune responses in the tobacco hornworm Manduca sexta causes increased susceptibility to the insect pathogen Photorhabdus. Dev Comp Immunol 2006;30:1099-1107.

48 Eleftherianos I, Boundy S, Joyce SA, Aslam S, Marshall JW, Cox RJ, Simpson TJ, Clarke DJ, ffrench-Constant RH, Reynolds SE: An antibiotic produced by an insect-pathogenic bacterium suppresses host defenses through phenoloxidase inhibition. Proc Natl Acad Sci USA 2007;104:2419-2424.

49 Eleftherianos I, Waterfield NR, Bone P, Boundy S, ffrench-Constant RH, Reynolds SE: A single locus from the entomopathogenic bacterium Photorhabdus luminescens inhibits activated Manduca sexta phenoloxidase. FEMS Microbiol Lett 2009;293:170176.

50 Ullah I, Khan AL, Ali L, Khan AR, Waqas M, Lee IJ, Shin JH: An insecticidal compound produced by an insect-pathogenic bacterium suppresses host defenses through phenoloxidase inhibition. Molecules 2014;19:2091320928.

51 Bode HB: Entomopathogenic bacteria as a source of secondary metabolites. Curr Opin Chem Biol 2009;13:224-230. 